\title{
Detection of a Far IR Excess with DIRBE at 60 and 100 Microns
}

D. P. Finkbeiner \& Marc Davis

Department of Astronomy, University of California, Berkeley, CA, 94720-3411, USA

\author{
David Schlegel \\ Princeton University, Princeton, NJ, USA
}

\begin{abstract}
From analysis of the DIRBE weekly averaged sky maps, we have detected substantial flux in the $60 \mu \mathrm{m}$ and $100 \mu \mathrm{m}$ channels in excess of expected zodiacal and Galactic emission (Finkbeiner, D.P., et al. 2000, ApJ, to be published, astro-ph/0004175). Two methods are used to separate zodiacal light from more distant emission. Both methods give consistent results at $60 \mu \mathrm{m}$ and $100 \mu \mathrm{m}$. The observed signal is consistent with an isotropic background of $\nu I_{\nu}=28.1 \pm 1.8 \pm 7$ (syst) $\mathrm{nW} \mathrm{m}^{-2} \mathrm{sr}^{-1}$ at $60 \mu \mathrm{m}$ and $24.6 \pm 2.5 \pm 8$ (syst) $\mathrm{nW} \mathrm{m}^{-2} \mathrm{sr}^{-1}$ at $100 \mu \mathrm{m}$.

The IR excess detected at 140 and $240 \mu \mathrm{m}$ by these methods agrees with previous measurements, which are thought to be the cosmic infrared background (CIB). The detections at 60 and $100 \mu \mathrm{m}$ are new. While this new excess is not necessarily the CIB, we have ruled out all known sources of emission in the solar system and Galaxy. We therefore tentatively interpret this signal as the CIB and consider the implications of such energy production from the viewpoint of star formation efficiency and black hole accretion efficiency. However, the IR excess exceeds limits on the CIB derived from the inferred opacity of the intergalactic medium to observed $\mathrm{TeV}$ photons, thus casting doubt on this interpretation. There is currently no satisfactory explanation for the $60-100 \mu \mathrm{m}$ excess.
\end{abstract}

